TRANSACTIONS OF THE

AMERICAN MATHEMATICAL SOCIETY

Volume 357, Number 9 , Pages 3775-3788

S 0002-9947(05)03691-3

Article electronically published on March 31, 2005

\title{
RENORMING JAMES TREE SPACE
}

\author{
PETR HÁJEK AND JAN RYCHTÁř
}

\begin{abstract}
We show that the James tree space $J T$ can be renormed to be Lipschitz separated. This negatively answers the question of J. Borwein, J. Giles and J. Vanderwerff as to whether every Lipschitz separated Banach space is an Asplund space.
\end{abstract}

\section{INTRODUCTION}

The main result of the present paper, Theorem 5 states the existence of an equivalent 2-WUR renorming (see Definition 2) of the James tree space JT. As a corollary, and this was in fact the motivation of our work, we answer in the negative a question of J. Borwein, J. Giles and J. Vanderwerff, whether every Lipschitz separated Banach space is an Asplund space.

Let us explain the situation in more detail. In [1] authors investigate properties of the Clarke subdifferential of a typical Lipschitz function on a given Banach space. In the course of their work, they study extensions of (bounded) Lipschitz functions from subspaces to the whole space, which preserve the Lipschitz constant. The results have implications for the behavior of the Clarke subdifferential. More precisely, they call a Banach space $(X,\|\|$.$) Lipschitz separated, if for every closed$ convex set $C \subset X$ and every bounded 1-Lipschitz real valued function $f$ on $C$ and $x \notin C$ there exist 1-Lipschitz extensions of $f$ on the whole $X$, say $f_{1}, f_{2}$, satisfying $f_{1}(x) \neq f_{2}(x)$. This property depends heavily on the norm $\|$.$\| . Let$ $B_{X}=\{x \in X ;\|x\| \leq 1\}$ be a unit ball. In [1] the following characterization is proved:

Theorem 1. For a given Banach space $(X,\|\|$.$) the following are equivalent:$

(1) $X$ is Lipschitz separated.

(2) For every pair of sequences $\left\{x_{n}\right\}_{n=1}^{\infty},\left\{y_{n}\right\}_{n=1}^{\infty} \subset B_{X}$ such that $\lim _{n, m \rightarrow \infty}\left\|x_{n}+y_{m}\right\|=2$, there is no $\phi \in X^{*}$ such that $\lim \sup _{n \rightarrow \infty} \phi\left(x_{n}\right)<0<\liminf _{n \rightarrow \infty} \phi\left(y_{n}\right)$.

It is observed that the WUR property of $\|$.$\| implies (2) and so does the 2-WUR$ (defined below), and on the other hand (2) implies that $\|.\|^{* *}$ is rotund. The last fact implies that $\ell_{1}$ is not isomorphic to any subspace of $X$.

Received by the editors October 3, 2003 and, in revised form, March 25, 2004.

2000 Mathematics Subject Classification. Primary 46B03.

Key words and phrases. Rotund norms, James tree space, Lipschitz separated Banach spaces.

The first author was supported by GAČR 201/01/1198 and A 10192 05. The second author was supported by GAČR 201/04/0090, GAUK 277/2001 and NSERC 7926.

(C)2005 American Mathematical Society Reverts to public domain 28 years from publication 
Definition 2. Let $(X,\|\|$.$) be a Banach space and let B_{X}$ be a closed unit ball. We say that the norm $\|\cdot\|$ is WUR (weakly uniformly rotund) if for all $f \in X^{*}$

$$
\lim _{n \rightarrow \infty} f\left(x_{n}-y_{n}\right)=0,
$$

whenever $\left\{x_{n}\right\}_{n=1}^{\infty},\left\{y_{n}\right\}_{n=1}^{\infty} \subset B_{X}$ are such that $\lim _{n \rightarrow \infty}\left\|x_{n}+y_{n}\right\|=2$. We say that $\|$.$\| is 2-WUR (2-weakly uniformly rotund) if for every f \in X^{*}$

$$
\lim _{n, m \rightarrow \infty} f\left(x_{n}-y_{m}\right)=0,
$$

whenever $\left\{x_{n}\right\}_{n=1}^{\infty},\left\{y_{n}\right\}_{n=1}^{\infty} \subset B_{X}$ are such that $\lim _{n, m \rightarrow \infty}\left\|x_{n}+y_{m}\right\|=2$.

Many examples of various renormings are presented in [1, supporting the natural conjecture that Lipschitz separated Banach spaces, although not necessarily WUR, should be WUR renormable or at least Asplund.

In our paper we construct a 2-WUR renorming $\|$.$\| of J T$, a classical example of a separable Banach space not containing $\ell_{1}$ and having non-separable dual. Thus $(J T,\|\|$.$) is Lipschitz separated, but is neither Asplund nor WUR renormable (see$ [5]). Recall that by [5], the space $J H$ of Hagler, which also does not contain $\ell_{1}$, does not admit an equivalent norm $\|$.$\| such that \|.\|^{* *}$ is rotund. Therefore $J H$ does not admit a Lipschitz separated renorming. Thus separable spaces with 2WUR renorming (or Lipschitz separating renorming) cut in between Asplund spaces and spaces not containing $\ell_{1}$. Thus, for separable Banach spaces, the property of being Lipschitz separated for some equivalent norm is strictly weaker than having a separable dual and strictly stronger than not containing $\ell_{1}$.

In this connection it would be interesting to find a rotundity renorming characterization of (separable) Banach spaces not containing $\ell_{1}$, similar to cases of superreflexive (UR), reflexive (2-R) or Asplund (WUR) (see [3], 7], [5]).

We also do not know whether $X$ admits an equivalent 2-WUR norm if $X$ is Lipschitz separated.

The organization of this paper is as follows. In Section 2 we introduce our notation and prove two lemmas that will be often used in the sequel. In Section 3 we state the main theorem and present the core of its proof. For the reader's convenience, proofs of auxiliary lemmas will be presented separately in Section 4 .

\section{Preliminaries And notation}

The James tree space $J T$ was introduced by J. Lindenstrauss and C. Stegall in [6]. Let us summarize the notation we use here. Let $T$ be an infinite dyadic tree, that is,

$$
T=\left\{(n, i) ; n \in \mathbb{N}, i \in\left\{0, \ldots, 2^{n}-1\right\}\right\} .
$$

We define a partial ordering $>$ on $T$ by letting $(m, j)>(n, i)$ if and only if $m>n$ and there is a sequence of integers $\left\{i_{k}\right\}_{k=0}^{m-n}$ such that $i_{0}=i, i_{m-n}=j$ and $i_{k} \in$ $\left\{2 i_{k-1}, 2 i_{k-1}+1\right\}$, for $k \leq m-n$. A maximal linearly ordered subset of $T$ will be called a branch. The set of all branches will be denoted $\mathcal{B}$. An interval $[s, t]$ is the maximal linearly ordered subset of $T$ with $s$ as a minimal element and $t$ as a maximal element. Similarly we define intervals $(s, t],(s, t),[s, t)$ and $[s, \infty)$. For every $t=(n, i) \in T$ we define the height of $t$ as $|t|=n$. Denote $T_{n}=\{t \in T ;|t| \leq$ $n\}$. For a non-empty and finite set $A \subset T$ define $\min (A)=\min \{|t| ; t \in A\}$ and 
$\max (A)=\max \{|t| ; t \in A\}$. For every real bounded function $x: T \rightarrow \mathbb{R}$ define a Hilbertian norm

$$
\left\|\left.x\left|\|^{2}=\sum_{t \in T} 2^{-4|t|}\right| x(t)\right|^{2} .\right.
$$

We say that $\mathcal{S}=\left(S_{j}\right)_{j=1}^{k}$ is an admissible collection if it is a collection of pairwise disjoint intervals of $T$. If $x: T \rightarrow \mathbb{R}$ is a real bounded function and $\mathcal{S}=\left(S_{j}\right)_{j=1}^{k}$ is an admissible collection, we define

$$
\|x\|_{\mathcal{S}}^{2}=\sum_{j=1}^{k}\left(\sum_{t \in S_{j}} x(t)\right)^{2}
$$

Observe that $\|\cdot\|_{\mathcal{S}}$ is a Hilbertian seminorm for every admissible collection $\mathcal{S}$. The James tree space $J T$ is defined in [6] as the space of all bounded functions $x: T \rightarrow \mathbb{R}$ such that $\|x\|_{J T}<\infty$, where

$$
\|x\|_{J T}^{2}=\sup \left\{\|x\|_{\mathcal{S}}^{2} ; \mathcal{S} \text { is an admissible collection }\right\} .
$$

Let us define an equivalent renorming $\|$.$\| of J T$ by means of the formula

$$
\|x\|^{2}=\|x\|_{J T}^{2}+\|x\|^{2} \text {. }
$$

Let $B_{J T}=\{x \in J T ;\|x\| \leq 1\}$. For $x \in J T$ we define the support of $x, \operatorname{supp}(x)$, by

$$
\operatorname{supp}(x)=\{t \in T ; x(t) \neq 0\} .
$$

For $\varepsilon>0$ and $x \in J T$ define

$$
\mathcal{A}(x, \varepsilon)=\left\{\mathcal{S} ; \mathcal{S} \text { is an admissible collection, }\|x\|_{\mathcal{S}}^{2}+\|x\|^{2}>\|x\|^{2}-\varepsilon^{2}\right\} .
$$

Observe that $\mathcal{A}(x, \varepsilon) \neq \emptyset$ for every $x \in J T$ and every $\varepsilon>0$.

If $\emptyset \neq S \subset T$ is a set, then we define $f_{S} \in J T^{*}$ by

$$
f_{S}(x)=\sum_{t \in S} x(t), \text { for every } x \in J T .
$$

The set $S$ will not always be finite but will be such that $f_{S}$ will always make sense. In case $S=\emptyset$ we set $f_{S} \equiv 0$. Note that if $S$ is an interval, then $\left\|f_{S}\right\| \leq 1$.

Let $x \in J T$ and $T^{\prime} \subset T$. We denote by $\left.x\right|_{T^{\prime}}$ the element of $J T$ such that $\left.x\right|_{T^{\prime}}(t)=x(t)$, for $t \in T^{\prime}$, and $\left.x\right|_{T^{\prime}}(t)=0$ for $t \notin T^{\prime}$.

Lemma 3. Let $\varepsilon_{0}>0, x, y \in B_{J T},\|x+y\|^{2}>4-\varepsilon_{0}^{2}$ and $\mathcal{S} \in \mathcal{A}\left(x+y, \varepsilon_{0}\right)$. Then

(i) $\mathcal{S} \in \mathcal{A}\left(x, \varepsilon_{0}\right) \cap \mathcal{A}\left(y, \varepsilon_{0}\right)$,

(ii) $\|x-y\|_{\mathcal{S}}^{2}<2 \varepsilon_{0}^{2}$.

Proof. By contradiction, let us assume that $\mathcal{S} \notin \mathcal{A}\left(x, \varepsilon_{0}\right)$. This implies that $\|x\|_{\mathcal{S}}^{2}+$ $\|x\|^{2} \leq 1-\varepsilon_{0}^{2}$. Using the parallelogram identity,

$$
\begin{aligned}
4-2 \varepsilon_{0}^{2} & <\|x+y\|^{2}-\varepsilon_{0}^{2}<\|x+y\|_{\mathcal{S}}^{2}+\|x+y\|^{2} \\
& \leq\|x+y\|_{\mathcal{S}}^{2}+\|x+y\|^{2}+\|x-y\|_{\mathcal{S}}^{2}+\|x-y\|^{2} \\
& =2\left(\|x\|_{\mathcal{S}}^{2}+\|x\|^{2}\right)+2\left(\|y\|_{\mathcal{S}}^{2}+\|y\|^{2}\right) \leq 2\left(1-\varepsilon_{0}^{2}\right)+2
\end{aligned}
$$


a contradiction. Hence $\|x\|^{2}-\varepsilon_{0}^{2}<\|x\|_{\mathcal{S}}^{2}+\|x\|^{2}$ and by the same argument $\|y\|^{2}-\varepsilon_{0}^{2}<\|y\|_{\mathcal{S}}^{2}+\|y\|^{2}$. Thus (i) is satisfied. Moreover

$$
\begin{aligned}
0 & \leq\|x-y\|_{\mathcal{S}}^{2}+\|x-y\|^{2} \\
& =2\left(\|x\|_{\mathcal{S}}^{2}+\|x\|^{2}\right)+2\left(\|y\|_{\mathcal{S}}^{2}+\|y\|^{2}\right)-\left(\|x+y\|_{\mathcal{S}}^{2}+\|x+y\|^{2}\right) \\
& \leq 2\|x\|^{2}+2\|y\|^{2}-\left(\|x+y\|^{2}-\varepsilon_{0}^{2}\right) \\
& <2+2-\left(4-2 \varepsilon_{0}^{2}\right)=2 \varepsilon_{0}^{2},
\end{aligned}
$$

and (ii) is satisfied.

Lemma 4. Let $x \in J T, \varepsilon_{0}>0$ and $\mathcal{S} \in \mathcal{A}\left(x, \varepsilon_{0}\right)$. Let $S \subset T$ be an interval such that $\mathcal{S}^{\prime}=\mathcal{S} \cup\{S\}$ is an admissible collection. Then

$$
\left|f_{S}(x)\right|<\varepsilon_{0}
$$

Proof.

$$
\begin{aligned}
\|x\|^{2} & \geq\|x\|_{\mathcal{S}^{\prime}}^{2}+\|x\|^{2}=\|x\|_{\mathcal{S}}^{2}+\left\|\left.x\left|\|^{2}+\right| f_{S}(x)\right|^{2}\right. \\
& >\|x\|^{2}-\varepsilon_{0}^{2}+\left|f_{S}(x)\right|^{2} .
\end{aligned}
$$

\section{MAIN THEOREM}

Theorem 5. The norm $\|$.$\| on JT defined above is 2-WUR.$

Proof. The proof proceeds by contradiction. Assume that there is $\varepsilon^{\prime}>0,\left\{x_{n}^{\prime}\right\}_{n=1}^{\infty}$, $\left\{y_{m}^{\prime}\right\}_{m=1}^{\infty} \in B_{J T}$ and $\varphi \in J T^{*}$ such that

$$
\lim _{n, m \rightarrow \infty}\left\|x_{n}^{\prime}+y_{m}^{\prime}\right\|=2
$$

and

$$
\limsup _{n \rightarrow \infty} \varphi\left(x_{n}^{\prime}\right)+\varepsilon^{\prime}<\liminf _{m \rightarrow \infty} \varphi\left(y_{m}^{\prime}\right) .
$$

In order to proceed faster to the core of the proof of Theorem 5, proofs of the following two facts are presented in the next section.

In our first step we replace $x_{n}^{\prime}, y_{m}^{\prime} \in J T$ and $\varphi \in J T^{*}$ by $x_{n}, y_{n} \in J T$ and $f_{B} \in J T^{*}$ having additional properties.

Fact 6. There is $\varepsilon \in\left(0,200^{-8}\right)$, and there are $x_{0} \in J T$, sequences $\left\{x_{k}\right\}_{k=1}^{\infty}$, $\left\{y_{l}\right\}_{l=1}^{\infty} \in B_{J T}, n_{0} \in \mathbb{N}$ and $B \in \mathcal{B}$ such that for all $k, l \in \mathbb{N}$

$$
f_{B}\left(x_{k}\right)+\sqrt[8]{\varepsilon} \leq f_{B}\left(y_{l}\right)
$$

and moreover

(a) $\left\|x_{k}+y_{l}\right\|>2-2 \varepsilon$,

(b) $\left.x_{k}\right|_{T_{n_{0}}}=\left.y_{l}\right|_{T_{n_{0}}}=x_{0}$,

(c) $n_{0}<\operatorname{mintail}\left(x_{k}\right)<\operatorname{maxtail}\left(x_{k}\right)<\operatorname{mintail}\left(y_{k}\right)$, for all $k \geq 1$,

(d) $\operatorname{mintail}\left(y_{l}\right)<\operatorname{maxtail}\left(y_{l}\right)<\operatorname{mintail}\left(x_{l+1}\right)$, for all $l \geq 1$,

where

$$
\begin{aligned}
& \operatorname{mintail}(x)=\min \left\{\operatorname{supp}\left(x-x_{0}\right)\right\} \\
& \operatorname{maxtail}(x)=\max \left\{\operatorname{supp}\left(x-x_{0}\right)\right\}
\end{aligned}
$$


From now on, $B$ will be the branch provided by Fact 6 . In the rest of the proof we will show that the statement of Fact [6 (i.e. the estimate (4)) is contradicting. Note that $x_{k}$ and $y_{l}$ are finitely supported for all $k, l \in \mathbb{N}$.

Fact 7. Upon passing to subsequences and keeping the original notation, there are sequences $\left\{x_{k}\right\}_{k=1}^{\infty},\left\{y_{l}\right\}_{l=1}^{\infty}$ and an admissible collection $\mathcal{S}_{k, l} \in \mathcal{A}\left(x_{k}+y_{l}, 9 \sqrt{\varepsilon}\right)$ such that the conclusion of Fact [6] still holds, and for all $k \in \mathbb{N}$ :

(a) $\min (S) \leq n_{k}$, for all $S \in \mathcal{S}_{k, l}$ and all $l \geq k$, where $n_{k}=\operatorname{maxtail}\left(x_{k}\right)$.

(b) For every $l \geq k$, every $S_{l} \in \mathcal{S}_{k, l}$ and every $l^{\prime} \geq k$, there is $S_{l^{\prime}} \in \mathcal{S}_{k, l^{\prime}}$ such that $S_{l} \cap T_{n_{k}}=S_{l^{\prime}} \cap T_{n_{k}}$. Moreover, if $S_{l}$ is such that $\max \left(S_{l}\right) \leq n_{k}$, then $S_{l}=S_{l^{\prime}}$

(c) If $P \subset T_{n_{k}}$ is a fixed set and $\mathcal{S}_{k, l}^{P} \subset \mathcal{S}_{k, l}$ is the collection of intervals starting at points of $P$, then traces

$$
\mathcal{S}_{k, l}^{P} \cap T_{n_{k}}:=\left\{S \cap T_{n_{k}} ; S \in \mathcal{S}_{k, l}^{P}\right\}
$$

are, for fixed $k \in \mathbb{N}$, independent of $l \geq k$. In particular, $\left\|x_{k}\right\|_{\mathcal{S}_{k, l}^{P}}$ are, for fixed $k \in \mathbb{N}$, independent of $l \geq k$.

Similarily, for all $l \in \mathbb{N}$ :

(d) $\min (S) \leq m_{l}$ for all $S \in \mathcal{S}_{k, l}$ and all $k>l$, where $m_{l}=\operatorname{maxtail}\left(y_{l}\right)$.

(e) For every $k>l$, every $S_{k} \in \mathcal{S}_{k, l}$ and every $k^{\prime}>l$, there is $S_{k^{\prime}} \in \mathcal{S}_{k^{\prime}, l}$ such that $S_{k} \cap T_{m_{l}}=S_{k^{\prime}} \cap T_{m_{l}}$. Moreover, if $\max \left(S_{k}\right) \leq m_{l}$, then $S_{k}=S_{k^{\prime}}$.

(f) If $P \subset T_{m_{l}}$ is a fixed set and $\mathcal{S}_{k, l}^{P} \subset \mathcal{S}_{k, l}$ is the collection of intervals starting at points of $P$, then traces

$$
\mathcal{S}_{k, l}^{P} \cap T_{m_{l}}:=\left\{S \cap T_{m_{l}} ; S \in \mathcal{S}_{k, l}^{P}\right\}
$$

are independent of $k>l$. In particular, $\left\|y_{l}\right\|_{\mathcal{S}_{k, l}^{P}}$ are independent of $k>l$.

Thus, by Lemma 3 .

$$
\left\|x_{k}-y_{l}\right\|_{\mathcal{S}_{k, l}}^{2}<2.9^{2} \varepsilon=162 \varepsilon,
$$

and consequently,

$$
\left|f_{S}\left(x_{k}-y_{l}\right)\right|^{2} \leq\left\|x_{k}-y_{l}\right\|_{\mathcal{S}_{k, l}}^{2}<162 \varepsilon
$$

for all $k, l \in \mathbb{N}$ and all $S \in \mathcal{S}_{k, l}$. The problem is that the branch $B$ need not be covered by intervals $S \in \mathcal{S}_{k, l}$ in a nice way to use (6) directly for estimating $\left|f_{B}\left(x_{k}-y_{l}\right)\right|$. Thus the following case analysis is needed.

For all $k, l \in \mathbb{N}$ we define subcollections $\mathcal{S}_{k, l}^{B}(i) \subset \mathcal{S}_{k, l}$, for $i=1,2,4,5$, of intervals starting on $B$ as follows:

$$
\begin{gathered}
\mathcal{S}_{k, l}^{B}(1)=\left\{S \in \mathcal{S}_{k, l} ; \min (S) \leq n_{0}, \max (S \cap B)<\operatorname{mintail}\left(x_{k}\right)\right\}, \\
\mathcal{S}_{k, l}^{B}(2)=\left\{S \in \mathcal{S}_{k, l} ; \min (S) \leq n_{0}, \max (S \cap B) \geq \operatorname{mintail}\left(x_{k}\right)\right\}, \\
\mathcal{S}_{k, l}^{B}(4)=\left\{S \in \mathcal{S}_{k, l} ; \min (S) \geq \operatorname{mintail}\left(x_{k}\right),\right. \\
\left.\max (S \cap B) \leq \operatorname{maxtail}\left(x_{k}\right)\right\} \\
\mathcal{S}_{k, l}^{B}(5)=\left\{S \in \mathcal{S}_{k, l} ; \min (S) \geq \operatorname{mintail}\left(x_{k}\right),\right. \\
\left.\max (S \cap B)>\operatorname{maxtail}\left(x_{k}\right)\right\} .
\end{gathered}
$$


Note that the above represents all intervals in $\mathcal{S}_{k, l}$ starting on $B$. In the following steps, we will define a partition of $B$ into at most six pieces $B_{k, l}^{0}, \ldots, B_{k, l}^{5}$ such that

$$
f_{B}\left(x_{k}-y_{l}\right)=\sum_{i=0}^{5} f_{B_{k, l}^{i}}\left(x_{k}-y_{l}\right),
$$

and we will estimate each term $\left|f_{B_{k, l}^{i}}\left(x_{k}-y_{l}\right)\right|$ separately.

The following proof is like an algorithm in the shape of a tree with seven levels. In first six levels, there is a branching in the proof. There are places where the proof ends, and there are two places where a certain estimate holds for all but finitely many $k \in \mathbb{N}$. This should be understood in the following way. If we come during the proof to such an estimate, we have to skip those finitely many $k \in \mathbb{N}$ for which that estimate does not hold, and after that we have to return to the beginning and start again at the Level 0 . If we come to the same estimate once more, it will already hold and we can proceed futher. For initiating the process, set $k=l=1$.

\section{Level 0.}

Set $m_{l}=\operatorname{maxtail}\left(y_{l}\right)$ and define $B_{k, l}^{0} \subset B \cap T_{m_{l}}$ as the maximal interval containing $(0,0)$ and such that $B_{k, l}^{0} \cap S=\emptyset$, for all $S \in \mathcal{S}_{k, l}$. Because $\mathcal{S}_{k, l} \cup B_{k, l}^{0}$ is an admissible collection, by Lemma 4

$$
\left|f_{B_{k, l}^{0}}\left(x_{k}-y_{l}\right)\right| \leq\left|f_{B_{k, l}^{0}}\left(x_{k}\right)\right|+\left|f_{B_{k, l}^{0}}\left(y_{l}\right)\right|<18 \sqrt{\varepsilon}
$$

If $B_{k, l}^{0}=B \cap T_{m_{l}}$, then the above inequality is a contradiction of (4) and the proof is finished. If $B_{k, l}^{0} \neq B \cap T_{m_{l}}$ we have to proceed to the next level.

\section{Level 1.}

Provided $S_{k, l}^{B}(1) \neq \emptyset$, let $B_{k, l}^{1}=\left[a_{1}, b_{1}\right] \subset B$ be an interval such that $a_{1}$ is the minimal and $b_{1}$ is the maximal element of $\left\{t \in B \cap S ; S \in \mathcal{S}_{k, l}^{B}(1)\right\}$. Set $B_{k, l}^{1}=\emptyset$ if $S_{k, l}^{B}(1)=\emptyset$. Because $x_{k}(t)=y_{l}(t)$ for all $t \in B_{k, l}^{1}$, we have that

$$
\left|f_{B_{k, l}^{1}}\left(x_{k}-y_{l}\right)\right|=0 \text {. }
$$

\section{Level 2.}

As $\mathcal{S}_{k, l}$ is an admissible collection, there is at most one interval in $\mathcal{S}_{k, l}^{B}(2)$. By Fact [7 the existence of such an interval does not depend on $l \geq k$. We will distinguish the following cases:

CAse I. There is $S_{k, l} \in \mathcal{S}_{k, l}^{B}(2)$ such that $\max \left(S_{k, l} \cap B\right) \geq \operatorname{maxtail}\left(x_{k}\right)$. Put

$$
\begin{aligned}
& S_{k, l}^{1}=\left(B \backslash S_{k, l}\right) \cap\left\{t \in T ;|t| \geq \operatorname{mintail}\left(y_{l}\right)\right\}, \\
& S_{k, l}^{2}=\left(S_{k, l} \backslash B\right) \cap\left\{t \in T ;|t| \geq \operatorname{mintail}\left(y_{l}\right)\right\} .
\end{aligned}
$$

By Fact 7 no interval of $\mathcal{S}_{k, l}$ starts after maxtail $\left(x_{k}\right)$ for $l \geq k$. Thus

$$
\mathcal{S}=\mathcal{S}_{k, l} \cup\left\{S_{k, l}^{1}\right\}
$$

is an admissible collection and by Fact 7 and Lemma 4

$$
\left|f_{S_{k, l}^{1}}\left(x_{k}-y_{l}\right)\right|=\left|f_{S_{k, l}^{1}}\left(y_{l}\right)\right|<9 \sqrt{\varepsilon} .
$$

By Lemma 11] there is $l \geq k$ such that

$$
\left|f_{S_{k, l}^{2}}\left(x_{k}-y_{l}\right)\right|=\left|f_{S_{k, l}^{2}}\left(y_{l}\right)\right|<20 \sqrt[4]{\varepsilon} .
$$


Finally, by (7) and (8)

$$
\begin{aligned}
\left|f_{B}\left(x_{k}-y_{l}\right)\right| \leq & \left|f_{B_{k, l}^{0}}\left(x_{k}-y_{l}\right)\right|+\left|f_{B_{k, l}^{1}}\left(x_{k}-y_{l}\right)\right|+\left|f_{S_{k, l}}\left(x_{k}-y_{l}\right)\right| \\
& +\left|f_{S_{k, l}^{1}}\left(x_{k}-y_{l}\right)\right|+\left|f_{S_{k, l}^{2}}\left(x_{k}-y_{l}\right)\right| \\
\leq & 18 \sqrt{\varepsilon}+0+9 \sqrt{\varepsilon}+9 \sqrt{\varepsilon}+20 \sqrt[4]{\varepsilon}
\end{aligned}
$$

a contradiction with (4). Thus the proof is finished.

CASE II. There is $S_{k, l} \in \mathcal{S}_{k, l}^{B}(2)$ such that

$$
\max \left(S_{k, l} \cap B\right)<\operatorname{maxtail}\left(x_{k}\right) \text { and } \max \left(S_{k, l}\right) \geq \operatorname{mintail}\left(y_{l}\right) .
$$

Let $B_{k, l}^{2}=S_{k, l} \cap B$ and $C_{k}=B_{k, l}^{2} \cap\left\{t \in T ;|t| \geq \operatorname{mintail}\left(x_{k}\right)\right\}$. By Fact $7 . C_{k}$ does not depend on $l \geq k$ and, by Lemma 10

$$
\left|f_{B_{k, l}^{2}}\left(x_{k}-y_{l}\right)\right|=\left|f_{C_{k}}\left(x_{k}\right)\right|<10 \sqrt[4]{\varepsilon}
$$

for all but finitely many $k \in \mathbb{N}$

CASE III. There is $S_{k, l} \in \mathcal{S}_{k, l}^{B}(2)$ such that

$$
\max \left(S_{k, l} \cap B\right)<\operatorname{maxtail}\left(x_{k}\right) \text { and } \max \left(S_{k, l}\right)<\operatorname{mintail}\left(y_{l}\right) .
$$

Let $B_{k, l}^{2}=S_{k, l} \cap B, A_{k}=S_{k, l} \cap T_{n_{0}}, C_{k}=B_{k, l}^{2} \cap\left\{t \in T ;|t| \geq \operatorname{mintail}\left(x_{k}\right)\right\}$. By Fact (7) $A_{k}$ and $C_{k}$ do not depend on $l \geq k$. By (6)

$$
\left|f_{S_{k, l}}\left(x_{k}-y_{l}\right)\right|=\left|f_{S_{k, l} \backslash A_{k}}\left(x_{k}\right)\right|<9 \sqrt{2 \varepsilon} .
$$

Thus, by Lemma 8

$$
\left|f_{B_{k, l}^{2}}\left(x_{k}-y_{l}\right)\right|=\left|f_{C_{k}}\left(x_{k}\right)\right|<10 \sqrt[4]{\varepsilon} .
$$

CASE IV. If $\mathcal{S}_{k, l}^{B}(2)=\emptyset$ set $B_{k, l}^{2}=\left(B \cap T_{n_{0}}\right) \backslash\left(B_{k, l}^{0} \cup B_{k, l}^{1}\right)$. By Fact [

$$
\left|f_{B_{k, l}^{2}}\left(x_{k}-y_{l}\right)\right|=0 \text {. }
$$

In the summary, after Level 2, the proof is either finished or we define $B_{k, l}^{2} \subset B$ such that, by (9), (10), and (11),

$$
\left|f_{B_{k, l}^{2}}\left(x_{k}-y_{l}\right)\right|<10 \sqrt[4]{\varepsilon}
$$

\section{Level 3.}

Set $m_{l}=\operatorname{maxtail}\left(y_{l}\right)$ and define $B_{k, l}^{3} \subset B \cap T_{m_{l}}$ as the maximal interval disjoint with $\left(B_{k, l}^{0} \cup B_{k, l}^{1} \cup B_{k, l}^{2}\right)$ and such that no interval of $\mathcal{S}_{k, l}$ starts on $B_{k, l}^{3}$. As in Level 0 ,

$$
\left|f_{B_{k, l}^{3}}\left(x_{k}-y_{l}\right)\right|<18 \sqrt{\varepsilon} .
$$

If $B \cap T_{m_{l}}=\bigcup_{i=0}^{3} B_{k, l}^{i}$ we have arrived at a contradiction with (4), otherwise we have to proceed further.

\section{Level 4.}

Provided $\mathcal{S}_{k, l}^{B}(4) \neq \emptyset$, set $B_{k, l}^{4}=\left[a_{4}, b_{4}\right] \subset B$ to be the interval such that $b_{4}$ is the maximal element of $\left\{t \in S \cap B ; S \in \mathcal{S}_{k, l}^{B}(4)\right\}$ and

$$
B_{k, l}^{4}=\left[(0,0), b_{4}\right] \backslash \bigcup_{i=0}^{3} B_{k, l}^{i} .
$$


If $S_{k, l}^{B}(4)=\emptyset$, set $B_{k, l}^{4}=\emptyset$. Assume that $J \in \mathcal{S}_{k, l}^{B}(4), J^{\prime} \in \mathcal{S}_{k^{\prime}, l}^{B}(4)$ are such that $J \cap J^{\prime} \neq \emptyset$. Then necessarily $J \cap J^{\prime} \cap B \neq \emptyset$. Thus $k=k^{\prime}$ and $J=J^{\prime}$. Hence, by Lemma 9, for all but finitely many $k \in \mathbb{N}$ and for all $l \geq k$,

$$
\left\|x_{k}\right\|_{\mathcal{S}_{k, l}^{B}(4)}^{2}<2 \cdot 18^{2} \varepsilon .
$$

Hence

and by Lemma 4

$$
\mathcal{S}_{k, l} \backslash \mathcal{S}_{k, l}^{B}(4) \in \mathcal{A}\left(x_{k},\left(9^{2} \varepsilon+2 \cdot 18^{2} \varepsilon\right)^{1 / 2}\right),
$$

$$
\left|f_{B_{k, l}^{4}}\left(x_{k}-y_{l}\right)\right|=\left|f_{B_{k, l}^{4}}\left(x_{k}\right)\right| \leq\left(9^{2} \varepsilon+2 \cdot 18^{2} \varepsilon\right)^{1 / 2}<100 \sqrt{\varepsilon} .
$$

\section{Level 5.}

This level is almost identical to Level 2, Case I. By the admissibility of set $\mathcal{S}_{k, l}$, there is at most one interval in $\mathcal{S}_{k, l}^{B}(5)$. By Fact 7 , its existence does not depend on $l \geq k$. If there is $S_{k, l} \in \mathcal{S}_{k, l}^{B}(5)$, put

$$
\begin{aligned}
& S_{k, l}^{1}=\left(B \backslash S_{k, l}\right) \cap\left\{t \in T ;|t| \geq \operatorname{mintail}\left(y_{l}\right)\right\}, \\
& S_{k, l}^{2}=\left(S_{k, l} \backslash B\right) \cap\left\{t \in T ;|t| \geq \operatorname{mintail}\left(y_{l}\right)\right\} .
\end{aligned}
$$

By Fact 7 no interval of $\mathcal{S}_{k, l}$ starts after maxtail $\left(x_{k}\right)$ for $l \geq k$. Thus

$$
\mathcal{S}=\mathcal{S}_{k, l} \cup\left\{S_{k, l}^{1}\right\}
$$

is an admissible collection and by Fact 7 and Lemma 4

$$
\left|f_{S_{k, l}^{1}}\left(x_{k}-y_{l}\right)\right|=\left|f_{S_{k, l}^{1}}\left(y_{l}\right)\right|<9 \sqrt{\varepsilon} .
$$

By Lemma 11, there is $l \geq k$ such that

$$
\left|f_{S_{k, l}^{2}}\left(x_{k}-y_{l}\right)\right|=\left|f_{S_{k, l}^{2}}\left(y_{l}\right)\right|<11 \sqrt[4]{\varepsilon} .
$$

Thus by (7), (8), (12), (13), and (14)

$$
\begin{aligned}
\left|f_{B}\left(x_{k}-y_{l}\right)\right| \leq & \sum_{i=0}^{4}\left|f_{B_{k, l}^{i}}\left(x_{k}-y_{l}\right)\right|+\left|f_{S_{k, l}}\left(x_{k}-y_{l}\right)\right| \\
& \quad+\left|f_{S_{k, l}^{1}}\left(x_{k}-y_{l}\right)\right|+\left|f_{S_{k, l}^{2}}\left(x_{k}-y_{l}\right)\right| \\
\leq & \sqrt{\varepsilon}(18+0+18+100+9+9)+10 \sqrt[4]{\varepsilon}+11 \sqrt[4]{\varepsilon},
\end{aligned}
$$

a contradiction of (4). Thus the proof for this case is finished.

If $\mathcal{S}_{k, l}^{B}(5)=\emptyset$, we have to go to the last level.

\section{Level 6.}

Define $B_{k, l}^{5} \subset B$ as the maximal interval disjoint with $\bigcup_{i=0}^{4} B_{k, l}^{i}$ and such that $\max \left(B_{k, l}^{5}\right)=\operatorname{maxtail}\left(y_{l}\right)$. By Fact $7, \mathcal{S}_{k, l} \cup B_{k, l}^{5}$ is an admissible collection and thus, by Lemma 4

$$
\left|f_{B_{k, l}^{5}}\left(x_{k}-y_{l}\right)\right|<9 \sqrt{\varepsilon} .
$$

Thus by (17), (8), (12), (13), (14), and (15)

$$
\left|f_{B}\left(x_{k}-y_{l}\right)\right| \leq \sum_{i=0}^{5}\left|f_{B_{k, l}^{i}}\left(x_{k}-y_{l}\right)\right| \leq 50 \sqrt[4]{\varepsilon},
$$

a final contradiction of (4). 


\section{Auxiliary lemmas}

Proof of Fact 6. As $\left\{x_{n}^{\prime}\right\}_{n=1}^{\infty},\left\{y_{m}^{\prime}\right\}_{m=1}^{\infty} \subset B_{J T}$ are such that

$$
\lim _{n, m \rightarrow \infty}\left\|x_{n}^{\prime}+y_{m}^{\prime}\right\|=2 \text {, }
$$

by [2, Fact II.2.3 (ii)],

$$
\lim _{n \rightarrow \infty} f_{\{t\}}\left(x_{n}^{\prime}\right)=\lim _{n \rightarrow \infty} x_{n}^{\prime}(t)=\lim _{m \rightarrow \infty} y_{m}^{\prime}(t)=\lim _{m \rightarrow \infty} f_{\{t\}}\left(y_{m}^{\prime}\right),
$$

for all $t \in T$. Recall that $J T_{*}$, the predual of $J T$, can be represented as

$$
J T_{*}=\overline{\operatorname{span}}\left\{f_{\{t\}} ; t \in T\right\} .
$$

Thus there is $x_{0}^{\prime} \in J T$ such that

$$
w^{*}-\lim _{n \rightarrow \infty} x_{n}^{\prime}=x_{0}^{\prime}=w^{*}-\lim _{m \rightarrow \infty} y_{m}^{\prime} .
$$

Since

$$
J T^{*}=\overline{\operatorname{span}}\left\{\left\{f_{\{t\}} ; t \in T\right\} \cup\left\{f_{A} ; A \in \mathcal{B}\right\}\right\},
$$

by (31), there is $B \in \mathcal{B}$ such that

$$
\lim _{n \rightarrow \infty} f_{B}\left(x_{n}^{\prime}\right) \neq \lim _{m \rightarrow \infty} f_{B}\left(y_{m}^{\prime}\right) .
$$

After a possible passing to subsequences and keeping the original notation, we may assume that there is $\varepsilon^{\prime \prime}>0$ such that

$$
\varepsilon^{\prime \prime}+f_{B}\left(x_{n}^{\prime}\right) \leq f_{B}\left(y_{m}^{\prime}\right),
$$

for all $n, m \in \mathbb{N}$. Pick

$$
\varepsilon<\min \left\{10^{-12}\left(\varepsilon^{\prime \prime}\right)^{8}, 200^{-8}\right\},
$$

and assume $\left\|x_{n}^{\prime}+y_{m}^{\prime}\right\| \geq 2-\varepsilon / 3$, for all $m, n \in \mathbb{N}$. Set

$$
n_{0}=\min \left\{n \in \mathbb{N} ;\left\|\left.x_{0}^{\prime}\right|_{T_{n}}\right\|>\left\|x_{0}^{\prime}\right\|-\varepsilon / 3\right\}+1,
$$

and define

$$
x_{0}=\left.x_{0}^{\prime}\right|_{T_{n_{0}}} .
$$

By (16) and (17) it is possible to find sequences $\left\{\widetilde{x}_{k}\right\}_{k=1}^{\infty},\left\{\widetilde{y}_{l}\right\}_{l=1}^{\infty} \subset J T$ and sequences $\left\{n_{k}\right\}_{k=1}^{\infty}$ and $\left\{m_{l}\right\}_{l=1}^{\infty}$ of integers such that

(a) $\left\|\widetilde{x}_{k}-x_{n_{k}}^{\prime}\right\|<\varepsilon / 3$,

(b) $\left\|\widetilde{y}_{l}-y_{m_{l}}^{\prime}\right\|<\varepsilon / 3$

(c) $\left.\widetilde{x}_{k}\right|_{T_{n_{0}}}=x_{0}=\left.\widetilde{y}_{l}\right|_{T_{n_{0}}}$,

(d) $n_{0}<\operatorname{mintail}\left(\widetilde{x}_{k}\right)<\operatorname{maxtail}\left(\widetilde{x}_{k}\right)<\operatorname{mintail}\left(\widetilde{y}_{k}\right)$ for all $k \in \mathbb{N}$,

(e) mintail $\left(\widetilde{y}_{l}\right)<\operatorname{maxtail}\left(\widetilde{y}_{l}\right)<\operatorname{mintail}\left(\widetilde{x}_{l+1}\right)$ for all $l \in \mathbb{N}$.

Clearly, $\left\|\widetilde{x}_{k}+\widetilde{y}_{l}\right\| \geq 2-\varepsilon$, and $\left\|\widetilde{x}_{k}\right\| \leq 1+\varepsilon / 3,\left\|\widetilde{y}_{l}\right\| \leq 1+\varepsilon / 3$, for all $k, l \in \mathbb{N}$. Set $x_{k}=(1+\varepsilon / 3)^{-1} \widetilde{x}_{k}$, and $y_{l}=(1+\varepsilon / 3)^{-1} \widetilde{y}_{l}$. Thus $\left\|x_{k}\right\| \leq 1,\left\|y_{l}\right\| \leq 1$, and

$$
\left\|x_{k}+y_{l}\right\| \geq(2-\varepsilon)(1+\varepsilon / 3)^{-1}>2-2 \varepsilon,
$$

for all $k, l \in \mathbb{N}$. Moreover, by (17),

$$
f_{B}\left(x_{k}\right)+\sqrt[8]{\varepsilon}<f_{B}\left(x_{k}\right)+\left(\varepsilon^{\prime \prime}-2 \varepsilon / 3\right)(1+\varepsilon / 3)^{-1} \leq f_{B}\left(y_{l}\right) .
$$


Proof of Fact [7. Note that $\left\|x_{k}+y_{l}\right\|^{2}>(2-2 \varepsilon)^{2}>4-10 \varepsilon$. For $k, l \in \mathbb{N}$ choose an admissible collection $\widetilde{\mathcal{S}}_{k, l} \in \mathcal{A}\left(x_{k}+y_{l}, 4 \sqrt{\varepsilon}\right)$ and define

$$
\begin{aligned}
& \mathcal{S}_{k, l}=\left\{S \in \widetilde{\mathcal{S}}_{k, l} ; S \cap \operatorname{supp}\left(x_{k}\right) \neq \emptyset \text { and } S \cap \operatorname{supp}\left(y_{l}\right) \neq \emptyset\right\}, \\
& \mathcal{S}_{k, l}^{x}=\left\{S \in \widetilde{\mathcal{S}}_{k, l} ; S \cap \operatorname{supp}\left(x_{k}\right) \neq \emptyset \text { and } S \cap \operatorname{supp}\left(y_{l}\right)=\emptyset\right\}, \\
& \mathcal{S}_{k, l}^{y}=\left\{S \in \widetilde{\mathcal{S}}_{k, l} ; S \cap \operatorname{supp}\left(y_{l}\right) \neq \emptyset \text { and } S \cap \operatorname{supp}\left(x_{k}\right)=\emptyset\right\} .
\end{aligned}
$$

We may and do assume that

$$
\widetilde{\mathcal{S}}_{k, l}=\mathcal{S}_{k, l} \cup \mathcal{S}_{k, l}^{x} \cup \mathcal{S}_{k, l}^{y} .
$$

By Lemma 3(ii) applied for $\varepsilon_{0}=4 \sqrt{\varepsilon},\left\|x_{k}-y_{l}\right\|_{\widetilde{\mathcal{S}}_{k, l}}^{2}<32 \varepsilon$. Thus the following estimate holds for both $\mathcal{S}=\mathcal{S}_{k, l}^{x}$ and $\mathcal{S}=\mathcal{S}_{k, l}^{y}$ :

$$
\begin{aligned}
\sum_{S \in \mathcal{S}}\left(\sum_{t \in S}\left(x_{k}+y_{l}\right)(t)\right)^{2} & =\sum_{S \in \mathcal{S}}\left(\sum_{t \in S}\left(x_{k}-y_{l}\right)(t)\right)^{2} \\
& =\left\|x_{k}-y_{l}\right\|_{\mathcal{S}}^{2} \leq\left\|x_{k}-y_{l}\right\|_{\widetilde{\mathcal{S}}_{k, l}}^{2}<32 \varepsilon .
\end{aligned}
$$

Hence

$$
\begin{aligned}
\left\|x_{k}+y_{l}\right\|_{\mathcal{S}_{k, l}}^{2}+\left\|x_{k}+y_{l}\right\|^{2} & \geq\left\|x_{k}+y_{l}\right\|_{\widetilde{\mathcal{S}}_{k, l}}^{2}+\left\|x_{k}+y_{l}\right\|^{2}-64 \varepsilon \\
& \geq\left\|x_{k}+y_{l}\right\|^{2}-16 \varepsilon-64 \varepsilon .
\end{aligned}
$$

This shows that

$$
\mathcal{S}_{k, l} \in \mathcal{A}\left(x_{k}+y_{l}, 9 \sqrt{\varepsilon}\right) .
$$

Clearly, $\mathcal{S}_{k, l}$ satisfies properties (a) and (d). Denote $E_{1}=\mathbb{N}$ and $k_{1}=\min \left(E_{1}\right)$. Set $n_{1}=\max \left(\operatorname{supp}\left(x_{1}\right)\right)$. There are only finitely many possibilities of what collections

$$
\mathcal{S}_{k_{1}, l} \cap T_{n_{1}}=\left\{S \cap T_{n_{1}} ; S \in \mathcal{S}_{k_{1}, l}\right\}
$$

can look like for $l \in E_{1}$. Thus there is an infinite set $E_{1}^{\prime} \subset E_{1}$ such that the system $\mathcal{S}_{k_{1}, l} \cap T_{n_{1}}$ does not depend on $l \in E_{1}^{\prime}$ and the first part of the property (b) is satisfied. For every $l \geq k_{1}$ and every interval $I \in \mathcal{S}_{k_{1}, l} \cap T_{n_{1}}$, there is a unique interval $I_{l} \in \mathcal{S}_{k_{1}, l}$ such that $I=I_{l} \cap T_{n_{1}}$. There are exactly two posibilities: either $\max \left(I_{l}\right)$ is less than mintail $\left(y_{l}\right)$ or it is not. Thus there is an infinite set $E_{1}^{\prime \prime} \subset E_{1}^{\prime}$ such that the above property is independent of $l \in E_{1}^{\prime \prime}$. Take $l_{1}=\min \left(E_{1}^{\prime \prime}\right)$. Assume that $k_{i}, l_{i}, E_{i}$ and $E_{i}^{\prime \prime}$ have been defined for $i=1, \ldots, N$. Define $E_{N+1}=$ $E_{N} \backslash\left\{k_{i}, l_{i} ; i=1, \ldots, N\right\}, k_{N+1}=\min \left(E_{N+1}\right)$ and repeat the above procedure. This will define sets of indexes $K=\left\{k_{i} ; i \in \mathbb{N}\right\}$ and $L=\left\{l_{i} ; i \in \mathbb{N}\right\}$ such that the properties (a) and (b) are satisfied for $k \in K$ and $l \in L, l \geq k$. Clearly, (c) follows from (b).

By the similar procedure, we get the properties (d), (e) and (f).

Lemma 8. Let $x \in B_{J T}, \varepsilon>0$ and $\mathcal{S} \in \mathcal{A}(x, 9 \sqrt{2 \varepsilon})$. Assume $S \in \mathcal{S}$ and an interval $A \subset S$ are such that

$$
\left|f_{S \backslash A}(x)\right|<18 \sqrt{2 \varepsilon} .
$$

Then

$$
\left|f_{C}(x)\right|<10 \sqrt[4]{\varepsilon}
$$

for any interval $C \subset S \backslash A$. 
Proof. Clearly $\mathcal{S}^{\prime}=\mathcal{S} \cup\{A, C\} \backslash\{S\}$ is an admissible collection. Thus

$$
\begin{aligned}
\|x\|^{2} & \geq\|x\|_{\mathcal{S}^{\prime}}^{2}+\|x\|^{2} \\
& =\|x\|_{\mathcal{S}}^{2}+\|x\|^{2}+\left|f_{A}(x)\right|^{2}+\left|f_{C}(x)\right|^{2}-\left|f_{S}(x)\right|^{2} \\
& =\|x\|_{\mathcal{S}}^{2}+\|x\|^{2}+\left|f_{A}(x)\right|^{2}+\left|f_{C}(x)\right|^{2}-\left|f_{S \backslash A}(x)+f_{A}(x)\right|^{2} \\
& \geq\|x\|^{2}-(9 \sqrt{\varepsilon})^{2}+\left|f_{C}(x)\right|^{2}-2\left|f_{S \backslash A}(x)\right|\left|f_{A}\left(x_{k}\right)\right|-\left|f_{S \backslash A}(x)\right|^{2} \\
& \geq\|x\|^{2}-81 \varepsilon-2.18 \sqrt{2 \varepsilon}-2.18^{2} \varepsilon+\left|f_{C}(x)\right|^{2} .
\end{aligned}
$$

Lemma 9. Let $\mathcal{S}_{k, l}^{0} \subset \mathcal{S}_{k, l}$, for $k, l \in \mathbb{N}$, be collections of intervals such that

$$
\mathcal{S}=\bigcup_{k \leq M} \mathcal{S}_{k, l}^{0}
$$

is an admissible collection for any $M \in \mathbb{N}$ and any $l \geq M$. Then

$$
\operatorname{card}\left\{k \in \mathbb{N} ;\left\|x_{k}\right\|_{\mathcal{S}_{k, l}^{0}}>18 \sqrt{2 \varepsilon} \text { for some } l \geq k\right\}<\left(\varepsilon^{-1 / 2}+1\right)^{2} .
$$

Proof. Put $N=\left(\varepsilon^{-1 / 2}+1\right)^{2}$. Note that $\left\|x_{k}\right\|_{\mathcal{S}_{k, l}^{0}}$ is independent of $l$ for $l \geq k$ by Fact 7 . Assume, by a contradiction, that the statement is not true. Without loss of generality we may assume that

$$
\left\|x_{k}\right\|_{\mathcal{S}_{k, l}^{0}}>18 \sqrt{2 \varepsilon}
$$

for all $k \leq N$ and $l=N$. By (5) and the triangle inequality,

$$
9 \sqrt{2 \varepsilon} \geq\left\|x_{k}-y_{l}\right\|_{\mathcal{S}_{k, l}} \geq\left\|x_{k}-y_{l}\right\|_{\mathcal{S}_{k, l}^{0}} \geq\left|\left\|x_{k}\right\|_{\mathcal{S}_{k, l}^{0}}-\left\|y_{l}\right\|_{\mathcal{S}_{k, l}^{0}}\right|,
$$

for all $k \leq N$. Therefore

$$
\left\|y_{l}\right\|_{\mathcal{S}_{k, l}^{0}}>9 \sqrt{2 \varepsilon}
$$

for all $k \leq N$. Hence

$$
1 \geq\left\|y_{l}\right\|^{2} \geq\left\|y_{l}\right\|_{\mathcal{S}}^{2}=\sum_{k=1}^{N}\left\|y_{l}\right\|_{S_{k, l}^{0}}^{2}>N(9 \sqrt{2 \varepsilon})^{2}>9^{2},
$$

a contradiction.

Lemma 10. Let $S_{k, l} \in \mathcal{S}_{k, l}^{B}(2)$ be such that

$$
\max \left(S_{k, l} \cap B\right)<\operatorname{maxtail}\left(x_{k}\right) \text { and } \max \left(S_{k, l}\right) \geq \operatorname{mintail}\left(y_{l}\right) .
$$

Let $A_{k}=S_{k, l} \cap T_{n_{0}}$ and $C_{k}=S_{k, l} \cap B \cap\left\{t \in T ;|t| \geq \operatorname{mintail}\left(x_{k}\right)\right\}$. Denote

$$
Q=\left\{k \in \mathbb{N} ;\left|f_{S_{k, l} \backslash A_{k}}\left(x_{k}\right)\right|>18 \sqrt{2 \varepsilon}, \text { for some } l \geq k\right\} .
$$

Then

$$
\operatorname{card}(Q)<\left(\varepsilon^{-1 / 2}+1\right)^{2}
$$

and for $k \notin Q$,

$$
\left|f_{C_{k}}\left(x_{k}\right)\right|<10 \sqrt[4]{\varepsilon}
$$


Proof. Note that, by Fact 7. $A_{k}, C_{k}$, and $f_{S_{k, l} \backslash A_{k}}\left(x_{k}\right)$ do not depend on $l \geq k$. Put $N=\left(\varepsilon^{-1 / 2}+1\right)^{2}$. Assume, by contradiction, that $\operatorname{card}(Q) \geq N$ and without loss of generality assume that $\{1, \ldots, N\} \subset Q$. Set $l=N+1$. Let $R_{k, l}=S_{k, l} \cap\{t \in$ $\left.T ;|t| \geq \operatorname{mintail}\left(y_{l}\right)\right\}$. Thus by (6)

$$
9 \sqrt{2 \varepsilon} \geq\left|f_{S_{k, l}}\left(x_{k}-y_{l}\right)\right|=\left|f_{S_{k, l} \backslash A_{k}}\left(x_{k}\right)-f_{R_{k, l}}\left(y_{l}\right)\right|,
$$

and consequently

$$
\left|f_{R_{k, l}}\left(y_{l}\right)\right|>9 \sqrt{2 \varepsilon}
$$

As $\mathcal{S}=\bigcup_{k=1}^{N} R_{k, l}$ is an admissible collection we have

$$
1 \geq\left\|y_{l}\right\|^{2} \geq\left\|y_{l}\right\|_{\mathcal{S}}^{2}=\sum_{k=1}^{N}\left|f_{R_{k, l}}\left(y_{l}\right)\right|^{2} \geq N(9 \sqrt{2 \varepsilon})^{2}>9^{2},
$$

a contradiction. Finally, by Lemma 8

$$
\left|f_{C_{k}}\left(x_{k}\right)\right|<10 \sqrt[4]{\varepsilon}
$$

for $k \notin Q$.

Lemma 11. Let $k_{0} \in \mathbb{N}$ be fixed. Upon passing to a subsequence of $\left\{y_{l}\right\}$,

$$
\left|f_{S_{k_{0}, l}^{2}}\left(y_{l}\right)\right|<11 \sqrt[4]{\varepsilon}
$$

for all $l \geq k_{0}$, where $S_{k_{0}, l}^{2}=\left(S_{k_{0}, l} \backslash B\right) \cap\left\{t \in T ;|t| \geq \operatorname{mintail}\left(y_{l}\right)\right\}$ and $S_{k_{0}, l} \in \mathcal{S}_{k_{0}, l}^{B}(2)$ or $S_{k_{0}, l} \in \mathcal{S}_{k_{0}, l}^{B}(5)$.

Proof. The proof will be divided into several steps. For simplicity of notation we will omit the index $k_{0}$ and write $L_{l}=S_{k_{0}, l}^{2}$. Notice that the family $\left\{L_{l} ; l \geq k_{0}\right\}$ is disjoint. Moreover

$$
\mathcal{S}:=\bigcup_{l \geq k_{0}}^{M}\left\{S \in \mathcal{S}_{k, l} ; S \text { starts on } L_{l}\right\}
$$

is an admissible collection for every $M \geq k_{0}$ and every $k>M$. Set $\mathcal{S}_{k, l}\left(L_{l}\right)=\{S \in$ $\mathcal{S}_{k, l} ; S$ starts on $\left.L_{l}\right\}$. By repeating the proof of Lemma 9 we get

$$
\operatorname{card}\left\{l \geq k_{0} ;\left\|y_{l}\right\|_{S_{k, l}\left(L_{l}\right)}>18 \sqrt{2 \varepsilon} \text { for some } k>l\right\}<\left(\varepsilon^{-1 / 2}+1\right)^{2} .
$$

Thus we may assume that

$$
\left\|y_{l}\right\|_{S_{k, l}\left(L_{l}\right)} \leq 18 \sqrt{2 \varepsilon}
$$

for all $l \in \mathbb{N}$ and all $k>l$. Hence $\mathcal{S}_{k, l} \backslash \mathcal{S}_{k, l}\left(L_{l}\right) \in \mathcal{A}\left(y_{l}, \sqrt{809 \varepsilon}\right)$. Define $L_{l}^{2} \subset L_{l}$ as the maximal interval such that, for all $k>l$, all intervals of $\mathcal{S}_{k, l}\left(L_{l}\right)$ start at some point of $L_{l}^{2}$. Notice that, by Fact 7, the definition of $L_{l}^{2}$ does not depend on $k>l$ and $\mathcal{S}_{k, l} \backslash \mathcal{S}_{k, l}\left(L_{l}\right) \cup\left\{L_{l}^{2}\right\}$ is an admissible collection. By Lemma 4 , we have

$$
\left|f_{L_{l}^{2}}\left(y_{l}\right)\right| \leq \sqrt{809 \varepsilon}<30 \sqrt{\varepsilon}
$$

Set $L_{l}^{1}=L_{l} \backslash L_{l}^{2}$. We claim that upon passing to a subsequence of $\left\{y_{l}\right\}$

$$
\left|f_{L_{l}^{1}}\left(y_{l}\right)\right|<10 \sqrt[4]{\varepsilon}
$$

for all $l \in \mathbb{N}$. Indeed, if $L_{l}^{1}=\emptyset$, the above inequality is trivial. If $L_{l}^{1} \neq \emptyset$, by the maximality of $L_{l}^{2}$, there has to be an interval $I_{k, l} \in \mathcal{S}_{k, l}$ such that $L_{l}^{1} \subset I_{k, l}$. We will distinguish the following two cases. By Fact 7 if one of them occurs for one $k>l$, then it occurs for all $k>l$. 
CASe I. $\max \left(I_{k, l}\right) \leq \operatorname{maxtail}\left(y_{l}\right)$. By Fact [7, $I_{k, l}$ does not depend on $k>l$. Put $I_{l}^{0}=I_{k, l} \cap T_{n_{0}}$. Thus, by (6), for $k>l+1$,

$$
\left|f_{I_{k, l} \backslash I_{l}^{0}}\left(y_{l}\right)\right|=\left|f_{I_{k, l}}\left(x_{k}-y_{l}\right)\right|<9 \sqrt{2 \varepsilon} .
$$

Since $L_{l}^{1} \subset I_{k, l} \backslash I_{l}^{0}$, by Lemma 8 ,

$$
\left|f_{L_{l}^{1}}\left(y_{l}\right)\right|<10 \sqrt[4]{\varepsilon} .
$$

CASE II. $\max \left(I_{k, l}\right) \geq \operatorname{mintail}\left(x_{k}\right)$. In this case the interval $I_{k, l}$ depends on $k>l$. Define $I_{k, l}^{0}=I_{k, l} \cap T_{n_{0}}, I_{k, l}^{1}=I_{k, l} \backslash I_{k, l}^{0}$, and $I_{k, l}^{2}=I_{k, l} \cap\left\{t \in T ; \operatorname{mintail}\left(x_{k}\right) \leq|t| \leq\right.$ $\left.\operatorname{maxtail}\left(x_{k}\right)\right\}$. Then

$$
\begin{gathered}
f_{I_{k, l}}\left(y_{l}\right)=f_{I_{k, l}^{0}}\left(x_{0}\right)+f_{I_{k, l}^{1}}\left(y_{l}\right), \\
f_{I_{k, l}}\left(x_{k}\right)=f_{I_{k, l}^{0}}\left(x_{0}\right)+f_{I_{k, l}^{2}}\left(x_{k}\right) .
\end{gathered}
$$

Thus, by (6),

$$
\left|f_{I_{k, l}^{1}}\left(y_{l}\right)-f_{I_{k, l}^{2}}\left(x_{k}\right)\right|=\left|f_{I_{k, l}}\left(x_{k}-y_{l}\right)\right|<9 \sqrt{2 \varepsilon} .
$$

We claim that

$$
\left|f_{I_{k, l}^{1}}\left(y_{l}\right)\right|<18 \sqrt{2 \varepsilon}
$$

for all $l>k_{0}$ and any $k>l$. Note that, by Fact 7 the above is independent of $k>l$. Indeed, if, for some $l_{0}>k_{0}$, the inequality (21) does not hold, then

$$
\left|f_{I_{k, l}^{2}}\left(x_{k}\right)\right|>9 \sqrt{2 \varepsilon}
$$

for all $k>l$. Set $k_{1}=l_{0}+1+\left(\varepsilon^{-1 / 2}+1\right)^{2}$. The family

$$
\mathcal{S}=\bigcup_{k=l_{0}+1}^{k_{1}} I_{k, l}^{2}
$$

is an admissible collection and

$$
1 \geq\left\|x_{k_{1}}\right\|^{2} \geq\left\|x_{k_{1}}\right\|_{\mathcal{S}}^{2}=\sum_{k=l_{0}+1}^{k_{1}}\left|f_{I_{k, l}^{2}}\left(x_{k}\right)\right|^{2}=\left(\varepsilon^{-1 / 2}+1\right)^{2}(9 \sqrt{\varepsilon})^{2}>9^{2},
$$

a contradiction.

Since $L_{l}^{1} \subset I_{k, l}^{1}$, by Lemma 8

$$
\left|f_{L_{l}^{1}}\left(y_{l}\right)\right|<10 \sqrt[4]{\varepsilon}
$$

Thus by (19) and (20)

$$
\left|f_{L_{l}}\left(y_{l}\right)\right| \leq\left|f_{L_{l}^{1}}\left(y_{l}\right)\right|+\left|f_{L_{l}^{2}}\left(y_{l}\right)\right| \leq 11 \sqrt[4]{\varepsilon} .
$$

\section{ACKNOWLEDGEMENTS}

The second author thanks Texas A\&M University for their hospitality while finishing this project. 


\section{REFERENCES}

[1] J. Borwein, J. Giles And J. VAnderwerfF: Rotund norms, Clarke subdifferentials and extensions of Lipschitz functions, Nonlinear Analysis 48, 2002, 287-301. MR 1870757 (2002h:49024)

[2] R. Deville, G. Godefroy and V. Zizler: Smoothness and renormings in Banach spaces, Pitman Monographs and Surveys in Pure and Applied Mathematics 64, Longman, 1993. MR.1211634 (94d:46012)

[3] P. ENFLO: Banach spaces which can be given an equivalent uniformly convex norm, Israel J. Math. 13, 1972, 281-288. MR0336297 (49:1073)

[4] M. Fabian, P. Habala, P. Hájek, V. Montesinos, J. Pelant and V. Zizler: Functional analysis and infinite dimensional geometry, Canadian Math. Soc. Books (Springer-Verlag), 2001. MR 1831176 (2002f:46001)

[5] P. HÁseK: Dual reormings of Banach spaces, Comment. Math. Univ. Carolinae 37, 1996, 241-554. MF,1398999 (97h:46013)

[6] J. Lindenstrauss AND C. Stegall: Examples of separable spaces which do not contain $\ell_{1}$ and whose duals are nonseparable, Studia Math. 54, 1975, 81-105. MR0390720 (52:11543)

[7] E. Odell ANd T. Schlumprecht: On asymptotic properties of Banach spaces under renormings, J. Amer. Math. Soc. 11, 1998, 175-188. MR.1469118 (98h:46006)

Mathematical Institute, AV ČR, Žitná 25, 11567 Praha 1, Czech Republic

E-mail address: hajek@math.cas.cz

Department of Mathematical and Statistical Sciences, University of Alberta, Edmonton, Alberta, Canada T6G 2G1

E-mail address: jrychtar@math.ualberta.ca

Current address: Department of Mathematical Sciences, University of North Carolina, Greensboro, North Carolina 27402 